\title{
Metallicities of the SPB stars from the IUE ultraviolet spectra ${ }^{\star}$
}

\author{
E. Niemczura ${ }^{\star}$
}

Astronomical Institute of the Wrocław University, ul. Kopernika 11, 51-622 Wrocław, Poland

Received 31 January 2003 / Accepted 8 April 2003

\begin{abstract}
We derived the stellar parameters (angular diameters, effective temperatures, metallicities) and interstellar reddenings for 20 SPB and 34 reference stars observed during the IUE satellite mission. The parameters were derived by means of an algorithmic procedure of fitting theoretical flux distributions to the low-resolution IUE spectra and optical spectrophotometric observations. Since the metallicity $[\mathrm{m} / \mathrm{H}]$ has a special importance for pulsating B type stars, we focused our attention on that parameter. We found that the mean value of the metallicity of the considered SPB and reference stars amounts to $[\mathrm{m} / \mathrm{H}] \approx$ -0.20 . The results only slightly depend on the reduction procedure used for the IUE images (NEWSIPS and INES). The metal abundances obtained in this paper are in accordance with the average value of -0.2 dex for stars in the solar neighborhood recently reported by other investigators.
\end{abstract}

Key words. stars: early-type - stars: abundances - stars: variables: general

\section{Introduction}

Knowledge of abundances of the iron-group elements in B-type stars is important for several reasons. In particular, a huge number of absorption lines of these elements causes the origin of the opacity bump at temperature around $2 \times 10^{5} \mathrm{~K}$. The pulsations of $\beta$ Cephei and Slowly Pulsating B (SPB) stars are driven by the classical $\kappa$-mechanism acting in this metal opacity bump (Dziembowski \& Pamyatnykh 1993; Gautchy \& Saio 1993). Consequently, the position of the instability domains in the Hertzsprung-Russell (H-R) diagram is sensitive to the metallicity parameter (Pamyatnykh 1999).

The ultraviolet (UV) part of the spectra of main-sequence B-type stars is very rich in lines of the iron-group elements. In this paper we analyze the IUE (International Ultraviolet Explorer, Bogges et al. 1978) data of the known SPB stars. The SPB stars have been discovered by Waelkens \& Rufener (1985) as a result of systematic observations of a selected sample of stars in the Geneva photometric system (see also Waelkens 1987, 1991). These main-sequence stars are characterized essentially by spectral types between B3 and B8 and pulsate in high-order low-degree $g$-modes (Waelkens 1991). Their pulsation periods range from about 1 to 3 days (De Cat et al. 2000). At present many SPB stars are known (Molenda-Żakowicz 2002; Waelkens et al. 1998). Twenty of them have IUE spectra of good quality. These spectra can be used to derive the main stellar parameters including the metallicity parameter,

\footnotetext{
* Tables 3-7 are only available in electronic form at the CDS via anonymous ftp to cdsarc.u-strasbg.fr $(130.79 .128 .5)$ or via http://cdsweb.u-strasbg.fr/cgi-bin/qcat?J/A+A/404/689 $\star \star$ e-mail: eniem@astro.uni.wroc.pl
}

$[\mathrm{m} / \mathrm{H}]$. Until now the metal abundances were determined for only three SPB stars, HD 147392, HD 160762 and HD 215573 (see Sect. 6.1 for more informations).

Usefulness of the low-resolution IUE observations in studying $[\mathrm{m} / \mathrm{H}]$ simultaneously with the effective temperature, $T_{\text {eff }}$, surface gravity, $\log g$, microturbulent velocity, $v_{t}$, angular diameter, $\theta$, and interstellar reddening parameter, $E(B-V)$, was demonstrated by Fitzpatrick \& Massa (1999). From the IUE and optical data, Niemczura et al. (2002) determined the main parameters of the standard stars listed by Code et al. (1976). Daszyńska et al. (2002) applied the same method to a sample of $\beta$ Cephei stars. Aznar Cuadrado \& Jeffery (2001) obtained parameters $\left(T_{\text {eff }}, \theta, E(B-V)\right.$ and $\left.[\mathrm{He} / \mathrm{H}]\right)$ of sdB stars on the basis of the IUE observations. Recently, Morossi et al. (2002) used the IUE spectra, combined with visual spectrophotometric observations, to derive $[\mathrm{m} / \mathrm{H}], T_{\text {eff }}$ and $\theta$ for 53 stars in the solar neighborhood.

In this paper we combined the IUE spectra with optical spectrophotometric data to derive the atmospheric parameters of a sample of 20 SPB and 34 reference stars listed in Tables 1 and 2, respectively. Single stars with the spectral type between B3 and B8 and luminosity class between V and III were selected as reference stars. In Sect. 2 the spectrophotometric observations and their calibration are presented. The procedure of fitting theoretical spectra to the observations is briefly described in Sect. 3. The methods of surface gravity determinations are outlined in Sect. 4. The results and the analysis of uncertainties of the obtained parameters are discussed in Sect. 5. In that section we also study interparametric correlations. A discussion of the metallicity of the analyzed stars and 
Table 1. The list of SPB stars. The Hipparcos parallax, $\pi$, numbers of the IUE images used to construct the mean spectra, sources of the ground-based data and of the SPB classification (last column) are also given.

\begin{tabular}{|c|c|c|c|c|c|c|}
\hline HD & Spectral & & & IUE images & & \\
\hline number & type & $\pi$ [mas] & SWP camera & LWP camera & LWR camera & Ref. \\
\hline 1976 & B5 IV & $2.38 \pm 0.69$ & 04905,06250 & & 04221,05420 & $1, a, b$ \\
\hline 6417 & B3 III & $2.63 \pm 0.73$ & 25179 & 05309 & & $2, \mathrm{a}$ \\
\hline 21071 & B7 V & $5.41 \pm 0.79$ & 27039 & 27027 & & $2, \mathrm{a}, \mathrm{c}$ \\
\hline 25558 & B3 V & $3.12 \pm 0.96$ & 20595 & & 16510 & $3, \mathrm{c}$ \\
\hline 26326 & B5 IV & $4.49 \pm 0.78$ & 20586 & 07008 & & $2, \mathrm{c}, \mathrm{d}$ \\
\hline 27396 & B4 IV & $7.03 \pm 0.79$ & 03800 & & 03380 & $1, \mathrm{e}, \mathrm{f}$ \\
\hline 34798 & B5 IV/V & $4.12 \pm 0.81$ & 21787,24997 & 05274 & & $2, a, c$ \\
\hline 37151 & B8 V & $4.15 \pm 0.88$ & 27623 & 07585 & & $2, \mathrm{c}, \mathrm{h}$ \\
\hline 37332 & B5 V & $3.07 \pm 1.00$ & 27588,32289 & 07565 & & $2, \mathrm{a}, \mathrm{c}$ \\
\hline 74195 & B3 IV & $6.59 \pm 0.51$ & 21415 & 02187 & & $2, a, d$ \\
\hline 74560 & B3 IV & $6.80 \pm 0.46$ & 53219 & 29789 & & $3, \mathrm{~d}, \mathrm{i}$ \\
\hline 109026 & B5 V & $10.07 \pm 0.52$ & 51268 & 29060 & & $1, \mathrm{c}$ \\
\hline 147394 & B5 IV & $10.37 \pm 0.53$ & $\begin{array}{l}54344,54345,54346,54347 \\
54348,55793,55795,55796\end{array}$ & $30424,30426,30427,30428$ & & $3, \mathrm{k}, \mathrm{c}$ \\
\hline 160762 & B3 V/IV & $6.58 \pm 0.56$ & $\begin{array}{l}29818,29820,29952,29956, \\
29957\end{array}$ & $\begin{array}{l}\text { 09641, 09642, 09768, 09774, } \\
\text { 09776, } 09777\end{array}$ & & $3, \mathrm{a}, \mathrm{g}$ \\
\hline 177863 & B8 V & $4.50 \pm 1.05$ & 48407 & 26177 & & $2, a, d$ \\
\hline 181558 & B5 III/V & $4.80 \pm 0.82$ & 47819 & 25691 & & $2, \mathrm{~d}, \mathrm{i}$ \\
\hline 182255 & B6 III & $8.10 \pm 0.68$ & 19291 & 15325 & & $1, a, b$ \\
\hline 183133 & B2 IV & $2.25 \pm 0.81$ & 20857,51067 & 28387 & & $2, b, c$ \\
\hline 208057 & B3 V & $6.37 \pm 0.70$ & $31518,32189,33664,31187$ & 29221 & & $3, \mathrm{a}, \mathrm{c}$ \\
\hline 215573 & B6 IV & $7.35 \pm 0.47$ & $\begin{array}{l}13508,29798,29799,29800, \\
29801,29802,29803,29804, \\
29817\end{array}$ & 09626, 09629, 09632, 09639 & & $1, a, b, d$ \\
\hline
\end{tabular}

References for ground-based data: 1 - Alekseeva et al. (1997), 2 - Mermilliod et al. (1997), 3 - Breger (1976).

References for SPB classification: a - Molenda-Żakowicz (2002), b - Koen (2001), c - Waelkens et al. (1998), d - De Cat (2001), e - De Ridder et al. (1999), f - Chapellier et al. (1998), g - Chapellier et al. (2000), h - North \& Paltani (1994), i - Waelkens (1991), k - Masuda \& Hirata (2000).

Table 2. The list of reference stars. The Hipparcos parallax, $\pi$, number of IUE images used to construct the mean spectra and sources of the ground-based data (last column) are given.

\begin{tabular}{|c|c|c|c|c|c|c|c|c|c|}
\hline \multirow{2}{*}{$\begin{array}{c}\text { HD } \\
\text { number }\end{array}$} & \multirow[b]{2}{*}{$\pi[\mathrm{mas}]$} & \multicolumn{2}{|c|}{ IUE images } & \multicolumn{2}{|r|}{ HD } & \multicolumn{4}{|c|}{ IUE images } \\
\hline & & SWP & LWP/LWR & Ref. & number & $\pi[\mathrm{mas}]$ & SWP & LWP/LWR & Ref. \\
\hline 15371 & $6.17 \pm 0.60$ & 2 & 1 & 3 & 87015 & $2.06 \pm 0.82$ & 1 & 1 & 2 \\
\hline 17081 & $7.40 \pm 0.85$ & 1 & 1 & 3 & 87901 & $42.09 \pm 0.79$ & 7 & 4 & 3 \\
\hline 20365 & $6.18 \pm 0.66$ & 1 & 1 & 3 & 102232 & $4.17 \pm 0.72$ & 1 & 1 & 2 \\
\hline 22928 & $6.18 \pm 0.85$ & 9 & 10 & 3 & 105382 & $8.68 \pm 0.72$ & 2 & 2 & 3 \\
\hline 23288 & $9.75 \pm 1.05$ & 3 & 2 & 2 & 115823 & $8.24 \pm 0.67$ & 1 & 1 & 2 \\
\hline 23324 & $8.87 \pm 0.89$ & 1 & 1 & 2 & 116226 & $1.82 \pm 0.71$ & 1 & 2 & 2 \\
\hline 23338 & $8.75 \pm 1.08$ & 3 & 2 & 2 & 120315 & $32.39 \pm 0.74$ & 59 & 107 & 3 \\
\hline 25340 & $7.20 \pm 0.83$ & 3 & 1 & 2 & 129116 & $10.69 \pm 0.72$ & 1 & 1 & 2 \\
\hline 29335 & $3.85 \pm 0.78$ & 1 & 2 & 2 & 131120 & $8.49 \pm 0.76$ & 1 & 1 & 1 \\
\hline 29589 & $9.46 \pm 0.78$ & 3 & 2 & 2 & 135485 & $5.52 \pm 1.13$ & 2 & 2 & 2 \\
\hline 32630 & $14.87 \pm 0.74$ & 13 & 25 & 3 & 135742 & $20.38 \pm 0.87$ & 1 & 1 & 3 \\
\hline 35580 & $4.35 \pm 0.51$ & 7 & 6 & 2 & 164852 & $3.17 \pm 0.71$ & 5 & 2 & 2 \\
\hline 36935 & $2.20 \pm 1.02$ & 1 & 1 & 3 & 181869 & $19.20 \pm 0.99$ & 4 & 1 & 2 \\
\hline 63118 & $3.68 \pm 0.48$ & 3 & 4 & 2 & 184171 & $5.20 \pm 0.55$ & 1 & 13 & 2 \\
\hline 67536 & $2.61 \pm 0.46$ & 19 & 1 & 2 & 188665 & $5.11 \pm 0.49$ & 3 & 2 & 2 \\
\hline 74280 & $6.99 \pm 0.92$ & 7 & 1 & 2 & 204770 & $4.23 \pm 0.48$ & 1 & 1 & 2 \\
\hline 79447 & $6.53 \pm 0.47$ & 1 & 1 & 1 & 209952 & $32.16 \pm 0.82$ & 7 & 3 & 3 \\
\hline
\end{tabular}

References for ground-based data: 1 - Alekseeva et al. (1997), 2 - Mermilliod et al. (1997), 3 - Breger (1976). 
a comparison with previous determinations are given in Sect. 6 . Finally, conclusions are presented in Sect. 7.

\section{The observational data}

The observational material consists of the IUE observations obtained with the large and small apertures from both the long-wavelength (LWP and LWR, 1950-3200 $\AA$ ) and the short-wavelength (SWP, 1100-1950 $\AA$ ) cameras with high $(0.1-0.3 \AA)$ and low $(6-7 \AA)$ spectral resolutions. We used observations processed by two reduction packages: the IUE/NEWSIPS (New Spectral Image Processing System, Nichols \& Linsky 1996; Gahart et al. 1997) and IUE/INES (IUE Newly Extracted Spectra, Rodríguez Pascual et al. 1999; Gonzáles-Riestra et al. 2001 and references therein).

Data points with a flag indicating some data-quality problems were excluded from further analysis. Because of the presence of interstellar $\operatorname{Ly} \alpha$ absorption, the spectral region around Ly $\alpha$ line was omitted. The IUE observations for $\lambda>$ $3000 \AA$ were also excluded from the analysis because of the very low signal-to-noise ratio at these wavelengths. Thus, we used the IUE fluxes in the spectral range of 1300-3000 $\AA$. Whenever more than one spectrum was available for a star, the observations were co-added using a cross-correlation technique. The spectra were re-binned to the theoretical wavelength points.

Detailed comparison of the IUE low-dispersion spectra extracted by the INES and NEWSIPS procedures has been done by Schartel \& Skillen (1998). They found an excellent agreement in most cases. Main differences are seen for underexposed spectra and in strong spectral lines. In Fig. 1, a comparison of the NEWSIPS and INES spectra of the considered SPB stars is presented. The systematic differences appear in the spectral range between 1305 and $1320 \AA$ where several strong lines are located. There are also systematic differences for highresolution spectra extracted by the INES and NEWSIPS procedures. They, however, occur mainly at the wavelengths where the LWP/LWR and SWP spectra overlap. Because of the low quality of the spectra in this wavelength range, we excluded these data from the further analysis. Furthermore, some discrepancies are also seen in strong lines in the SWP spectra. Large differences occur in the wavelength range 2000-2300 for highly reddened stars. Other discrepancies between the IUE/NEWSIPS and IUE/INES data are probably not systematic (see Fig. 1). In general, better agreement between spectra extracted from both procedures exists for high-resolution data.

The ultraviolet observations expressed in the absolute units were supplemented by optical spectrophotometric measurements taken from the Breger Catalogue (Breger 1976) and Pulkovo Spectrophotometric Catalogue (Alekseeva et al. 1997). In addition, we used Johnson $U B V$ and Strömgren $u v b y$ magnitudes if no optical spectrophotometric data were available (see Tables 1 and 2). The magnitudes were converted into fluxes by means of the formula $F_{\lambda}=10^{0.4\left(C_{\lambda}-m_{\lambda}\right)}$, with the scaling factors $C_{\lambda}$ taken from Gray (1998) and Bessell (1979) for the $u v b y$ and $U B V$ filters, respectively. We also used the Geneva photometry to derive surface gravities of the stars. All photometric data used in this paper were taken from the GCPD database (General Catalogue of Photometric Data, Mermilliod et al. 1997). There is no Geneva photometry for HD 6417 and there are no uvby $\beta$ indices for HD 183133.

\section{The analysis}

We analyzed the low-resolution spectra by means of an algorithmic procedure of fitting the theoretical flux distributions, $f_{\lambda}^{\mathrm{t}}$, to the observations, $f_{\lambda}$. The theoretical flux can be written in the form:

$f_{\lambda}^{t}=(\theta / 2)^{2} \times F_{\lambda}(\boldsymbol{a}) \times 10^{-0.4 A_{\lambda}}$,

where $F_{\lambda}$ and $A_{\lambda}$ denote the surface flux and total interstellar extinction, respectively. The flux, $F_{\lambda}$, depends on several atmospheric parameters, shown here as a vector $\boldsymbol{a}=$ $\boldsymbol{a}\left(T_{\text {eff }}, \log g,[\mathrm{~m} / \mathrm{H}], v_{\mathrm{t}}\right)$. We used theoretical fluxes calculated by Kurucz (1996) with the standard value of the microturbulent velocity, $v_{\mathrm{t}}=2 \mathrm{~km} \mathrm{~s}^{-1}$, for all analyzed objects. The grid of Kurucz's model atmospheres covers $T_{\text {eff }}$ between 2000 and $200000 \mathrm{~K}, \log g$ from 0.0 to 5.0 and $[\mathrm{m} / \mathrm{H}]$ from -5.0 to 1.0. Theoretical fluxes are published at 1221 points in the wavelength range from $90 \AA$ to $160 \mu \mathrm{m}$, with a step of the order of $10 \AA$ in the UV, $50 \AA$ in the visual part of the spectrum, and increasing from 50 to $200 \AA$ in the infrared region. The spectral resolutions of the observational and theoretical data were adjusted before the analysis.

The total extinction along the line of sight, $A_{\lambda}$, can be written in the form $A_{\lambda}=E(B-V)[k(\lambda-V)+R(V)]$, where $k(\lambda-V)$ stands for the normalized extinction curve and $R(V)$ is the ratio of total to selective extinction. We adopted the mean interstellar reddening curve of Fitzpatrick (1999) for the majority of the analyzed stars. In some cases the mean curve is not a good approximation because of spatial variability of the extinction law. The effect will be larger for stars with high reddenings. Because of this we computed five additional parameters, specifying the shape of the UV extinction curve (Fitzpatrick 1999) for stars with $E(B-V)>0.10$ mag. Three of them describe the Lorentzian-like bump at $2200 \AA$ (its width, $\gamma$, position, $x_{0}$ and strength, $c_{3}$ ), one is the far-UV curvature term $\left(c_{4}\right)$ and the last one is the linear term $\left(c_{2}\right)$.

In this paper we also included two additional parameters, $S_{1}$ and $S_{2}$, which are used to adjust absolute levels of the three parts of the observed fluxes. The parameters $S_{1}$ and $S_{2}$ adjust the short- and long-wavelength parts of IUE spectra to the visual flux level. Outside the IUE/SWP region we put $S_{1}=1$, whereas $S_{2}=1$ is set outside the wavelength region covered by the long-wavelength IUE observations. Introducing these two parameters is justified by several reasons. First, for some stars we used the IUE observations made with a small aperture and these data are not calibrated to the absolute level with high precision. Second, pulsation effects can also introduce disagreements in the absolute levels of spectra obtained in different times (it has to be remembered that the amplitude of the pulsations of SPB stars increases with decreasing wavelength). Third, the SWP and LWP (or LWR) observations we used were sometimes made some years apart. In such cases, errors in absolute levels caused by instrumental effects can be significant. Finally, different absolute calibrations of the UV 


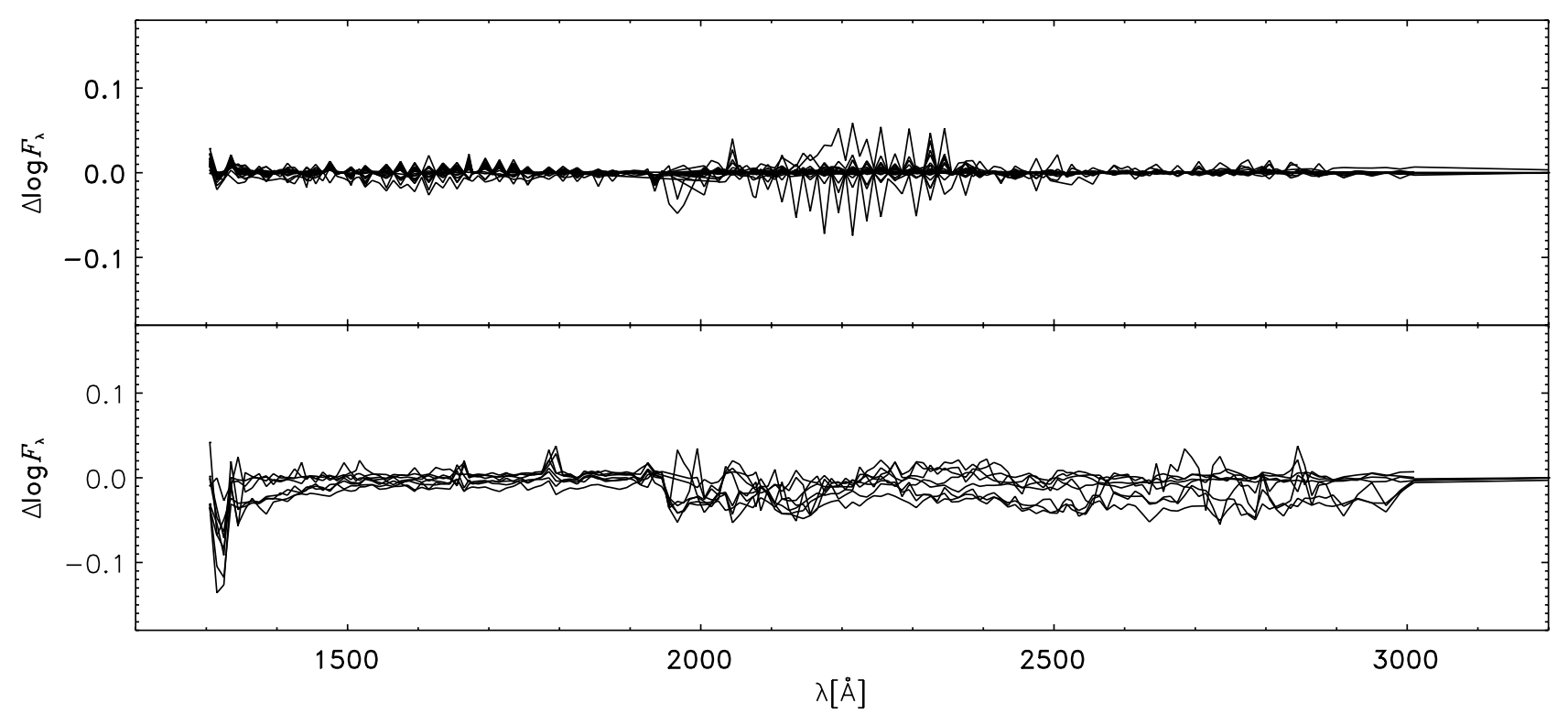

Fig. 1. Differences, $\Delta \log F_{\lambda}$, between the IUE/INES and the IUE/NEWSIPS high-resolution (top) and low-resolution (bottom) spectra of the SPB stars.

and visual parts of the spectrum may result in a difference of levels between these two spectral ranges. The parameters $S_{1}$ and $S_{2}$ were incorporated into the procedure as follows:

$\log \tilde{f}_{\lambda}^{\mathrm{t}}=\log f_{\lambda}^{\mathrm{t}}+\log S_{1}+\log S_{2}$.

In order to derive the vector of all parameters, we used the method of analysis based on the least-squares optimization algorithm (Bevington 1969, see also Fitzpatrick \& Massa 1999; Niemczura et al. 2002). The problem of simultaneous determination of all parameters during the best-fit procedure can be solved if the parameters produce detectable and different spectral signatures. Fitzpatrick \& Massa (1999) illustrated how the atmospheric parameters $\left(T_{\text {eff }}, \log g,[\mathrm{~m} / \mathrm{H}], v_{\mathrm{t}}\right)$ and $E(B-V)$ affect the spectrum of a star having $T_{\text {eff }}=20000 \mathrm{~K}, \log g=$ $4.0 \mathrm{dex},[\mathrm{m} / \mathrm{H}]=0.0 \mathrm{dex}$ and $v_{\mathrm{t}}=2 \mathrm{~km} \mathrm{~s}^{-1}$. They concluded that the spectral signatures produced by these parameters are different. One can therefore expect that the parameters could be obtained simultaneously by the best-fit procedure. In practice, however, $\log g$ is determined with a very high error, sometimes exceeding 1.0 dex (see Sect. 5.1 for more details). Owing to this we decided to determine $\log g$ by making use of the photometric calibrations, as well as the stellar evolutionary tracks. This approach is described in Sect. 4. The influence of the remaining parameters $\left(\theta, S_{1}, S_{2}\right)$ on the stellar flux is distinct from that of the stellar model parameters, $\boldsymbol{a}$. A more comprehensive analysis of the correlations between simultaneously determined parameters is presented in Sect. 5.1.

Finally, the vector of all parameters determined simultaneously in this paper, $\boldsymbol{p}$, can be expressed as:

$\boldsymbol{p}=\boldsymbol{p}\left(T_{\mathrm{eff}},[\mathrm{m} / \mathrm{H}], \theta, E(B-V), S_{1}, S_{2}\right)$.

For stars with $E(B-V)>0.10$ mag, five additional parameters were included:

$\boldsymbol{p}=\boldsymbol{p}\left(T_{\mathrm{eff}},[\mathrm{m} / \mathrm{H}], \theta, E(B-V), S_{1}, S_{2}, \gamma, x_{0}, c_{2}, c_{3}, c_{4}\right)$
We used the least-squares fitting method to determine the vector of parameters, $\boldsymbol{p}$, which minimizes the root mean square deviation, rms, defined as follows:

$\mathrm{rms}=\left[\sum_{i} \frac{\left(\log f_{i}-\log \tilde{f}_{i}^{\mathrm{t}}\right)^{2}}{\sigma_{i}^{2}}\right]^{1 / 2}$

where $\sigma_{i}^{2}$ are the variances of the observed fluxes, $\log f_{i}$, at the wavelength points $\lambda_{i}$. We adopted $\sigma_{i}^{2}=1$ for all wavelengths.

\section{Gravities of the program stars}

In this section we present the methods used to determination of the surface gravities of stars.

In the first one, gravities were derived using the Napiwotzki et al.'s (1993) version of the well-known code written by T. T. Moon and based on the two-dimensional $\left(\beta, c_{0}\right)$ grid published by Moon \& Dworetsky (1985). The errors of $\log g$ depend linearly on the effective temperature and range from $\approx 0.09$ dex for $T_{\text {eff }}=9000 \mathrm{~K}$ to $\approx 0.26 \mathrm{dex}$ for $T_{\text {eff }}=$ $30000 \mathrm{~K}$.

In the second method, gravities were determined by means of the relationships defined by Balona (1994). In this method the surface gravity and effective temperature are obtained independently. The formula for determining surface gravity of early-type stars leads to the standard deviation in $\log g$ of about 0.1 dex for a typical photometric error of 0.01 mag in $c_{0}$ and $\beta$. However the error quickly increases to $0.3 \mathrm{dex}$ and becomes even larger for hot stars with $\beta<2.60$ or $c_{0}<-0.18$.

In the third method we made use of the biparametric calibration constructed by Ribas et al. (1997). This calibration enables us to estimate surface gravities for the main-sequence stars using a photometric index related to the effective temperature $\left(c_{0}\right)$ and another one related to the stellar evolution $(\beta)$. Ribas et al. (1997) estimated that the mean residuals of the surface gravity derived in this way amount to about 0.08 dex. 


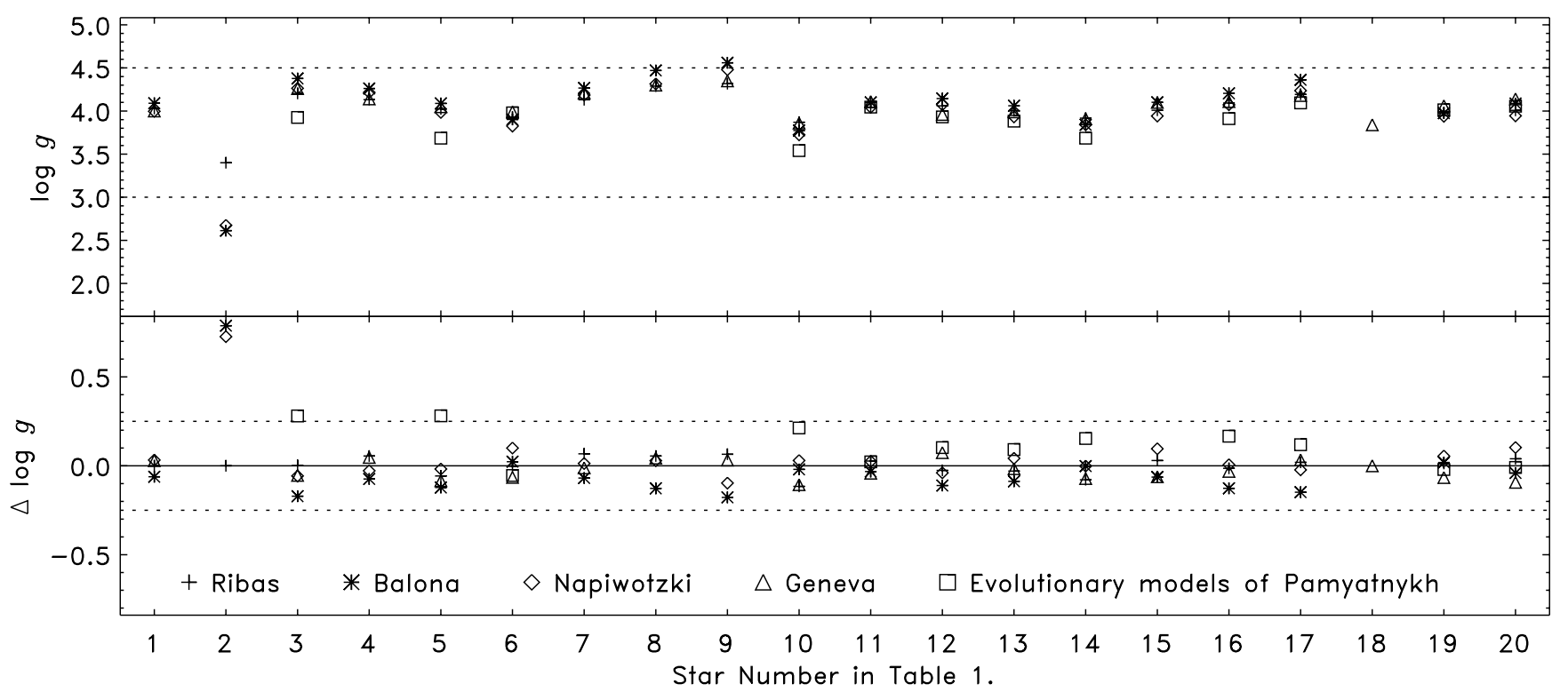

Fig. 2. Top: surface gravities for the SPB stars from different methods (see text). The dashed lines limit the values included to the analysis. Bottom: the differences in the sense $\Delta \log g=\log g_{\text {mean }}-\log g_{i}$. The dashed lines in the bottom panel denote $\Delta \log g= \pm 0.25$ dex.

The fourth approach uses the Geneva photometry. The efficiency of this approach is similar to that of the methods based on the $u v b y \beta$ system (see North \& Nicolet 1990; Kobi \& North 1990 and Künzli et al. 1997). In this paper we adopted the code written by Künzli et al. (1997), which allowed us to determine surface gravities together with errors, for a given metallicity. We used surface gravities for $[\mathrm{m} / \mathrm{H}]=0.0$ in the further analysis, because the differences between $\log g$ derived for different values of $[\mathrm{m} / \mathrm{H}]$ are within the error bars.

In the last method, we estimated the gravities using the formula obtained from stellar evolutionary models calculated by Pamyatnykh (1999) for OPAL opacities with $Z=0.02$. For this purpose we considered the main sequence evolutionary tracks of stars with masses of 3.0-6.5 $M_{\odot}$, with a step of $0.5 M_{\odot}$. We found the following relation:

$\log g=-12.4294+4.4628 \log T_{\text {eff }}-0.8208 \log L / L_{\odot}$.

The standard deviation for this equation amounts to 0.01 dex. We assumed that this formula can be applied to the investigated stars with the luminosity expressed as $\log L / L_{\odot}=2 \log R+$ $4 \log T_{\text {eff }}-2 \log R_{\odot}-4 \log T_{\text {eff }}^{\odot}$, where $R=\theta / 2 \pi$ is the stellar radius, $\pi$ is the stellar parallax, $T_{\text {eff }}^{\odot}$ and $R_{\odot}$ denote solar values of the effective temperature and radius, respectively. We used Hipparcos parallaxes (ESA 1997). During the best-fit procedure the current luminosity is corrected for the Lutz-Kelker bias (Lutz \& Kelker 1973, see also Jerzykiewicz \& MolendaŻakowicz 2000). The corrections for the luminosity can be calculated if $\sigma_{\pi} / \pi<0.175$. Consequently, we can determine $\log g$ by means of this method only for stars with $\sigma_{\pi} / \pi<0.175$ (see Tables 1 and 2). This method of determination of $\log g$ was included into the iteration process and the values of $T_{\text {eff }}$ and $\theta$ were calculated during the best-fit procedure. Two kinds of errors in surface gravities calculated from Eq. (6) can be important. The first one is introduced by stellar evolutionary models.
Systematic errors can be caused by physical ingredients incorporated in the computing code (e.g., overshooting, chemical composition). In particular, the overshooting is not taken into account in the evolutionary models, and we used the results obtained for $X=0.70, Y=0.28$, and $Z=0.02$. On the basis of the evolutionary models of Pamyatnykh (1999), we found that differences in $\log g$ are of the order of 0.01 dex when the formulas similar to Eq. (6), but corresponding to $Z=0.01$ and $Z=0.03$ are used with the same value of $X=0.70$.

Surface gravities of SPB stars calculated as described above are plotted in Fig. 2. In most cases, the values of $\log g$ derived by different methods agree quite well for both SPB and reference objects. The largest values of $\log g$ were typically found from Balona's (1994) method or from the Geneva photometry (Künzli et al. 1997). In most cases, the smallest surface gravities result from the method based on Eq. (6). The values of $\log g$ obtained for the same star can differ between each other by about 0.25 dex. A similar behaviour is seen for the reference stars.

It is important to know how uncertainties in $\log g$ influence the remaining parameters. To answer this question, we determined the stellar parameters $\boldsymbol{p}$ assuming $\log g_{i}$ from 3.50 to 4.50 with a step of 0.25 dex. The results are presented in Fig. 3, where $\Delta p_{j}=p_{j}\left(\log g_{i}\right)-p_{j}(\log g=4.0)$ is shown as a function of $\Delta \log g_{i}=\log g_{i}-4.0$. The index $i$ denotes the assumed value of $\log g$, whereas index $j$ stands for the individual parameter included in the vector $\boldsymbol{p}$. As one can see, there is little influence of $\Delta \log g_{i}$ on $\log T_{\text {eff }}, \theta, E(B-V), S_{1}$ and $S_{2}$ for the majority of stars even if $\Delta \log g_{i}=0.5$. However, for individual objects, the changes in parameters can be significant. The influence of $\log g$ on the metallicity parameter amounts typically to less than 0.20 dex, but can reach 0.40 dex for $\Delta \log g_{i}=0.50$. The underestimation of the surface gravity results in the lower metallicity parameter and vice versa. 

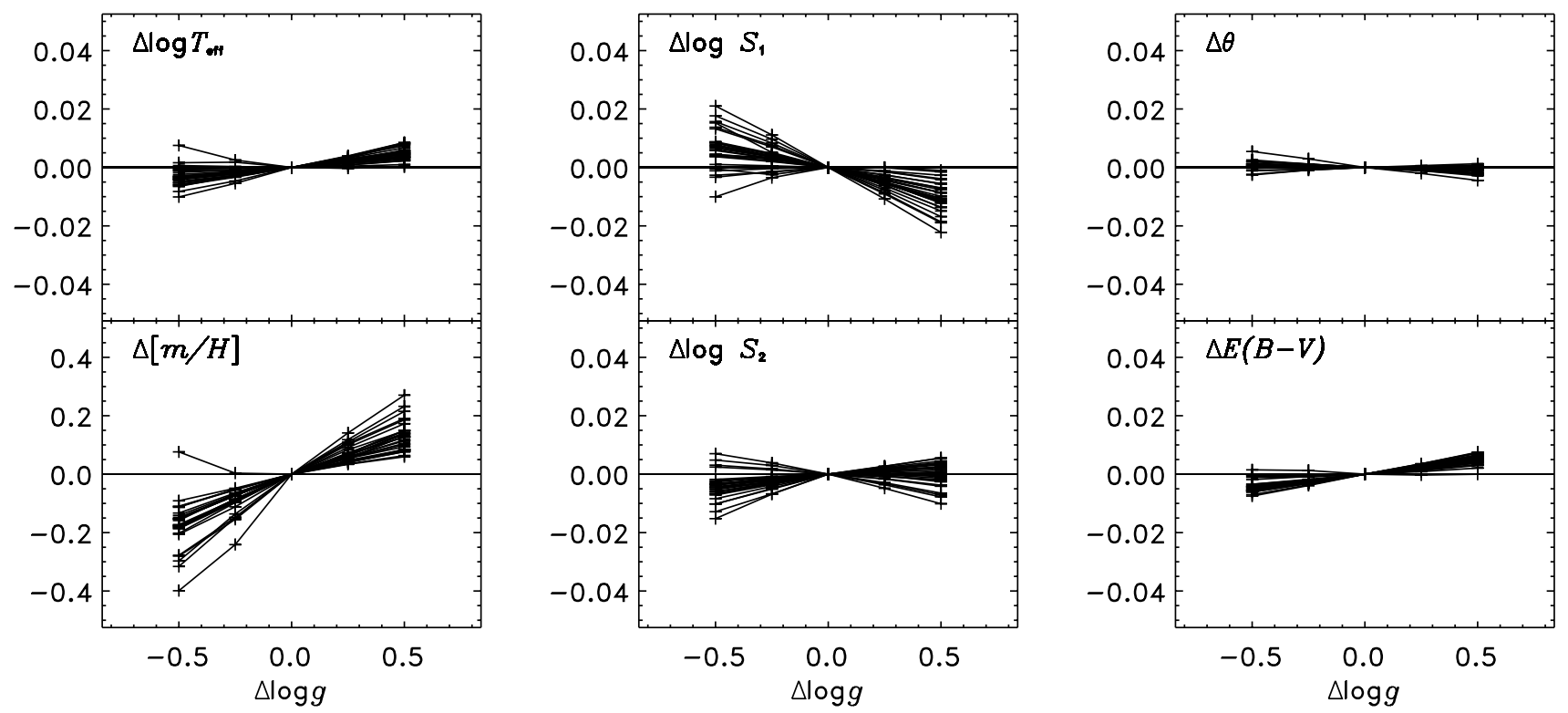

Fig. 3. The influence of the change of the surface gravity on the remaining parameters of the vector $\boldsymbol{p}$. The values of the parameters obtained for a given star are connected.

Owing to the effects discussed above we used the average value of $\log g$ for a given star. However, gravities lower than 3.00 dex and greater than 4.50 dex were excluded from the averaging process.

\section{Results}

Tables 3 and 4 show the parameters of the SPB stars, determined by using the IUE data calibrated by NEWSIPS and INES procedures, respectively. The tables have the same format and contain the best-fit solutions (upper line) and the results of a bootstrap resampling method described below (lower line). The best-fit parameters of reference stars obtained on the basis of both IUE calibrations packages are shown in Table 5. Figure 4 presents an example of a comparison of the IUE and optical observations (points) with the best-fit models (lines). There is a good overall agreement between these two data sets. Most of the analyzed stars have small interstellar reddenings. Only ten stars have $E(B-V)>0.10 \mathrm{mag}$, and for nine of them we determined individual extinction curves, parametrized by the five additional parameters, given in Table 6 for both calibrations. In case of the reference star HD 67536 this procedure did not give satisfactory results because of low quality of the IUE/LWP spectrum. For comparison, parameters of the standard extinction curve are also shown in Table 6.

In Fig. 5 we compare two sets of parameters obtained by using data calibrated by NEWSIPS and INES procedures. We find a good overall agreement for all parameters. In particular, the mean value of the difference, $\left(\Delta \log T_{\text {eff }}\right)_{\text {mean }}=$ $\left(\log T_{\text {eff }}(\text { NEWSIPS })-\log T_{\text {eff }}(\text { INES })\right)_{\text {mean }}=0.007 \pm 0.001 \mathrm{dex}$ (standard deviation $\sigma=0.008 \mathrm{dex}$ ). Similarly insignificant differences were found for $E(B-V), \theta$ and $[\mathrm{m} / \mathrm{H}]$ with the mean values equal to $0.005 \pm 0.001 \mathrm{mag}(\sigma=0.009 \mathrm{mag})$, $0.004 \pm 0.001 \mathrm{mas}(\sigma=0.007 \mathrm{mas})$ and $0.07 \pm 0.01 \mathrm{dex}$ $(\sigma=0.06 \mathrm{dex})$, respectively. The mean differences are rather small in all cases, but the standard deviations are larger. This means that for individual stars the differences can be significant. The largest differences in the parameters are obtained for stars with at most two spectra available from each IUE camera.

The values of the parameters $\log S_{1}$ and $\log S_{2}$ are typically lower than 0.10 in absolute units. The values of $\log S_{2}$ higher than 0.10 were obtained for the small aperture IUE data (HD 21071). The factor $\log S_{1}$ higher than 0.10 was determined for HD 6417, where we have only photometric data in the optical part of the spectrum and a single IUE spectrum. In general, higher values of $\log S_{1}$ and $\log S_{2}$ are obtained for stars with single IUE observations. By adding the parameter $\log S_{1}$ we can adjust the ultraviolet part of the spectrum to the visual one. However, we can also adjust the optical part to the IUE/UV data. In the latter case we derive different values of the stellar diameters, but the differences are within the error bars for most stars.

The binarity of the stars can also affect the determined parameters. Among the SPB stars listed in Table 1, nine were mentioned in the literature as binary systems. Two of them, HD 34798 and HD 181558, are wide visual binaries with separations equal to 39.3 arcsec (Abt 1988) and 46.9 arcsec (Gahm et al. 1983), respectively. HD 1976 is a triple system in which separations between the main component and the two fainter companions are about $0.1-0.2$ arcsec (see for example Hartkopf et al. 2000) and 0.8 arcmin (Tokovinin 1997). The mass of the closer companion was estimated to be about $3 M_{\odot}$, compatible with a main-sequence A-type star (Tokovinin 1997). HD 74560 is a spectroscopic binary system of SB1 type. De Cat (2001) obtained orbital elements for this object and concluded that the secondary component is an A-type star with a mass in the range 2.0-3.0 $M_{\odot}$. HD 177863 is a double system with a separation of 0.7 arcsec. De Cat (2001) determined orbital elements of the system and the mass of the secondary component is $1.7-2.4 M_{\odot}$, again corresponding to an A-type star. In all these cases the companion is fainter. 


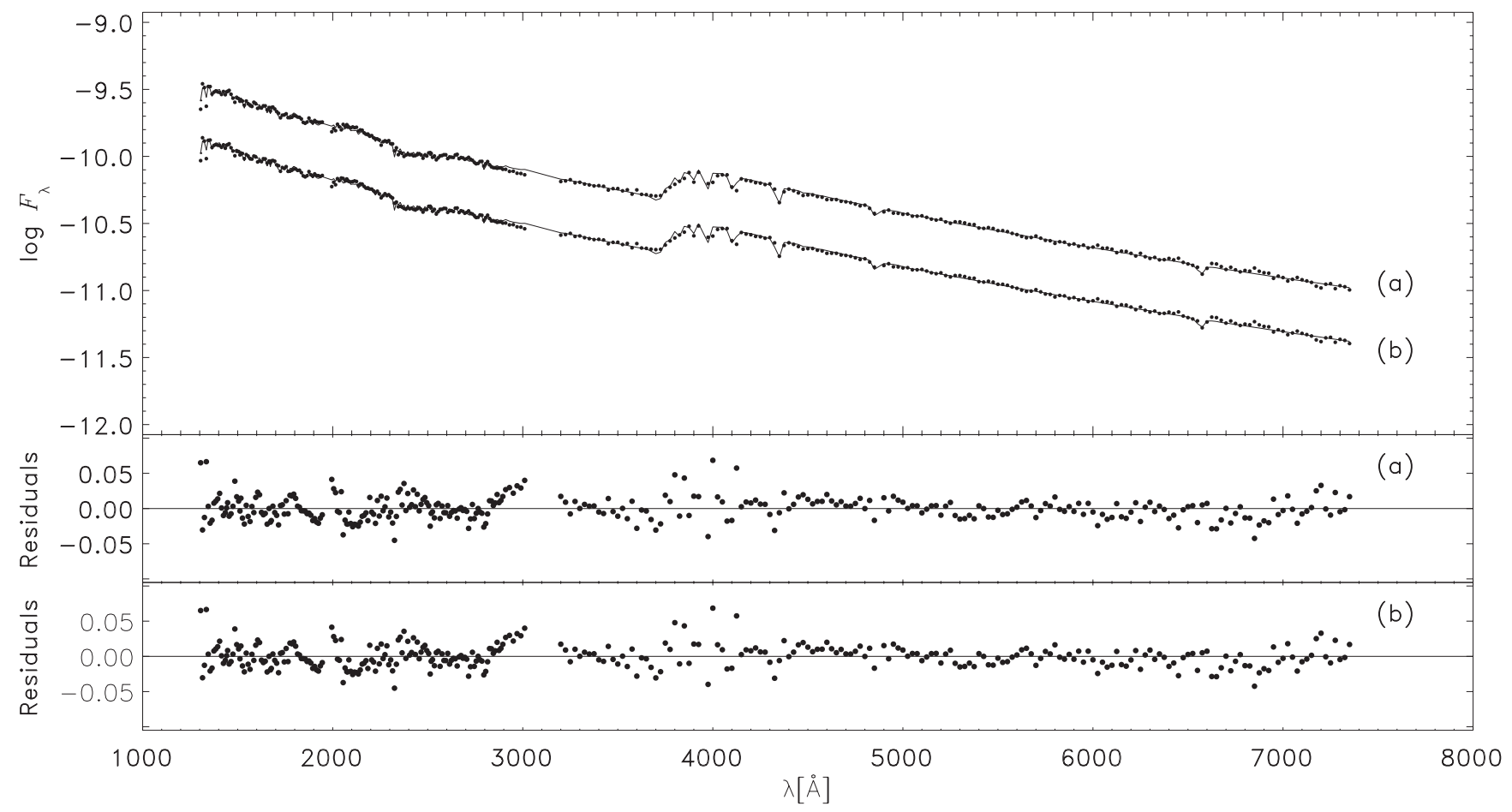

Fig. 4. Top: dereddened stellar energy distributions of HD 215573 (circles) compared with the best-fit models (solid lines) for the two calibrations of the ultraviolet IUE observations, NEWSIPS (a) and INES (b). The same optical data were used in both cases. For the INES data set an offset equal to -0.4 dex was applied for clarity. Bottom: the residuals from the two fits.
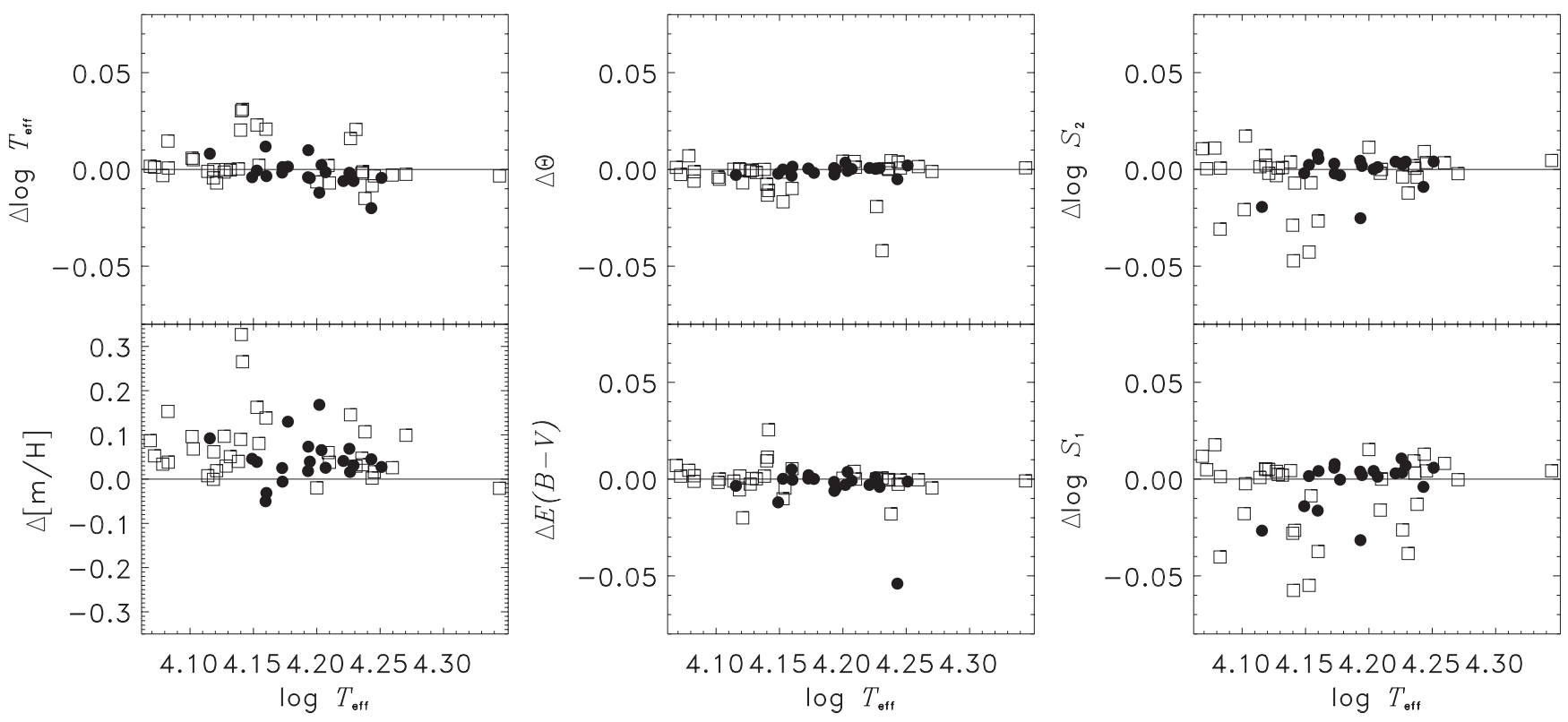

Fig. 5. Differences between best-fit parameters $\boldsymbol{p}$ in the sense $\Delta p_{i}=p_{i}$ (NEWSIPS) $-p_{i}$ (INES) plotted as a function of $\log T_{\text {eff }}$ for the SPB (filled circles) and reference stars (squares).

In addition, most of the flux of a main sequence A-type star is radiated in the visual part of the spectrum. For our procedure the ultraviolet part of the spectrum is crucial for parameter determination, hence the presence of an A-type (or later) companion(s) does not affect the results significantly. Four other objects, HD 74195, HD 182255, HD 183133 and HD 160762, were also mentioned as binary systems. According to De Cat (2001) HD 75195 is a binary system too close to be resolved (separation $<0.1$ arcsec) or with a very faint secondary component. HD 183133 was found to be a non-eclipsing, interacting binary with a period of $48.08 \mathrm{~d}$ (Lawson et al. 1994). Binarity of this object was not confirmed by other investigators and without knowledge of the secondary component it is hard to estimate its influence on the derived parameters. HD 182255 is an SB1 binary (Hube \& Aikman 1991) with a period of $367 \mathrm{~d}$ and a mass function of $0.0144 \pm 0.0042 M_{\odot}$. 


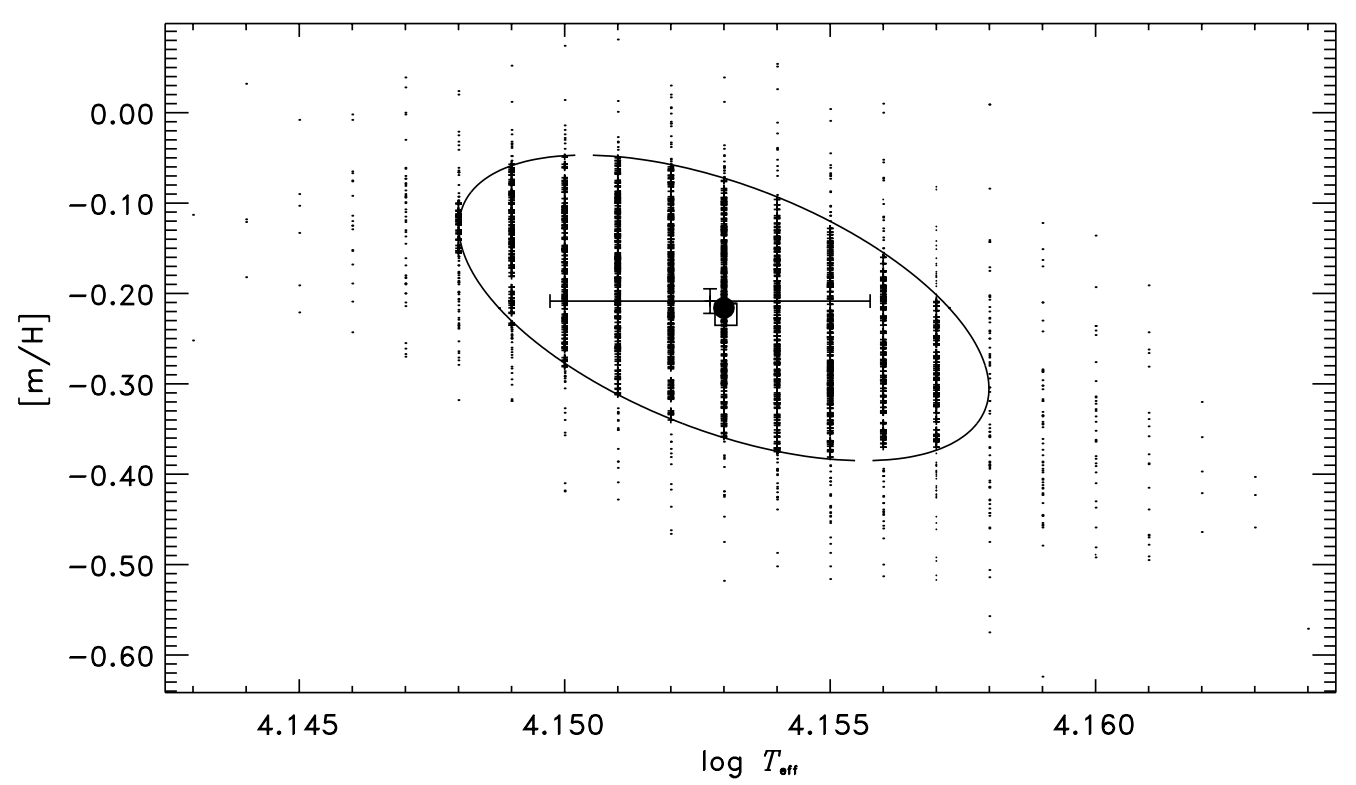

Fig. 6. Distribution of the effective temperature and metallicity obtained from the bootstrap method for HD 215573. The results shown as plus signs (included inside the ellipse) define the $1 \sigma$ uncertainty level by enclosing $68.3 \%$ of all cases. The best-fit solution is given with the error bars, whereas the mean value from the bootstrap sampling is plotted as a filled circle and the median value as a square.

HD 160762 is also an SB1 system, with a period of $113.804 \mathrm{~d}$. Abt \& Levy (1978) determined the orbital parameters of the system and the mass function of the order of $0.001369 M_{\odot}$. We do not expect that the binarity will affect the determined parameters since the secondary components are always much fainter and/or less massive stars.

\subsection{Uncertainties of the parameters}

The errors of best-fit parameters shown in Tables 3, 4, 5 and 6 are the formal rms errors resulting from the leastsquares method. These errors are definitely underestimated. The technique of bootstrap resampling is probably the most useful method for estimating of confidence levels for complex least-squares solutions (Press et al. 1992, Chap. 15.6, see also Maceroni \& Ruciński 1997). This technique uses the input data set, $D_{0}$, containing $N$ points to generate simulated data sets, $D_{i}$, with the same number of points. The symbol $i$ denotes the successive simulation. The number of all bootstrap simulations is equal to $N(\log N)^{2}($ Babu \& Singh 1983). In the generated data, the random fraction of original points $(37 \%$ in our case, Press et al. 1992, Chap. 15.6) are replaced by the remaining original points by means of random resampling with replacement. The simulated data sets are analyzed in the same way as the original data. This procedure makes it possible to estimate reliable uncertainties of the parameters and can be used for determining correlations between them. An example of the results of bootstrap resampling is plotted in Fig. 6. For each star the best-fit parameters are very close to the median and the mean values obtained from bootstrap resampling (see Tables 3, 4 and 6) and are well included in the contours, which were determined to enclose $68.3 \%$ of the bootstrap solutions around their median values. In most cases, the contours are elliptical in shape; they indicate correlations between the parameters. The uncertainties, defined as the one-sigma ranges around the median values, are definitively larger than the formal errors of the least-squares method (see Tables 3, 4 and 6).

The mean correlations $\left(\rho_{\text {mean }}\right)$, their standard deviations $\left(\sigma_{\rho}\right)$, median values $\left(\rho_{\mathrm{m}}\right)$ and ranges of correlation coefficients for the SPB stars are shown in Table 7. As one can see from the table, the correlations between metallicity and other parameters are rather small. However, the standard deviations, $\sigma_{\rho}$, are large. This means that for individual stars the values of $\rho$ can be significant. In most cases the strongest correlations are obtained for HD 182255. The median values clearly show that for most stars these correlations are small. Small values of $\rho_{\text {mean }}$ indicate that $[\mathrm{m} / \mathrm{H}]$ does not depend on the remaining parameters and can be determined from the best-fit procedure with good precision. The next parameter describing stellar atmosphere, the effective temperature, is more correlated with other parameters. The smallest value of $\rho_{\text {mean }}$ was obtained between $T_{\text {eff }}$ and $[\mathrm{m} / \mathrm{H}]\left(\rho_{\text {mean }}=0.25\right)$. Correlations between effective temperature and the remaining parameters are stronger and amount to about 0.65 . The smallest values were obtained for HD 34798 , HD 67536 and HD 74560, whereas the largest for HD 182255. The color excess is mainly correlated with the effective temperature $\left(\rho_{\text {mean }}=0.65\right)$. The remaining correlations are definitely smaller. The stellar diameter, $\theta$, is mainly correlated with $T_{\text {eff }}$ and $S_{2}$.

For the heavily reddened stars we obtained correlations of parameters describing the interstellar reddening curve. The parameter describing the location of the bump of the curve, $x_{0}$, is in fact independent of the remaining parameters. The largest value of $\rho_{\text {mean }}$ is between $x_{0}$ and $T_{\text {eff }}$; it is equal to 0.59 , with standard error $\sigma_{\rho}=0.18$. There are no meaningful correlations between $c_{4}$ and the remaining parameters: the largest values of $\rho_{\text {mean }}$ and $\rho_{\mathrm{m}}$ lie between 0.40 and 0.50 (see Table 7). The largest values of $\rho_{\text {mean }}$ occur for $c_{3}$ and the two other parameters describing the reddening curve, $E(B-V)$ and $c_{2}$. These 
values amount to 0.67 and 0.56 , respectively. In addition, $c_{2}$ is mainly correlated with $\theta, \rho_{\text {mean }}=0.61$. The last parameter describing the extinction curve, $\gamma$, does not depend on the other parameters.

We also applied the bootstrap resampling method to derive surface gravities. In most cases we did not obtain satisfactory results because the values of $\log g$ determined simultaneously with other stellar parameters are lower than photometric values by about 0.50 dex with the $1 \sigma$ uncertainties higher than $0.55 \mathrm{dex}$. So large values of $\sigma$ are caused by the broad range of $\log g$ determined by using the bootstrap resampling method in these cases. The surface gravities can range from less than 3.00 to more than 4.80 dex. For a few stars we obtained similar results from both methods, i.e., the determined values of $\log g$ were nearly the same as the photometric ones, with the $1 \sigma$ uncertainties equal to about $0.20-0.40$ dex. In these cases the other parameters in $\boldsymbol{p}$ are the same to within the formal errors, as the values determined with the photometric surface gravities. The correlations between $\log g$ and the remaining parameters depend on the star. When we obtain good values of the surface gravity, the correlations are lower than 0.50 (on the average $0.20-0.30$ ). In other cases the values of $\rho$ can be higher than 0.70 .

Because in the majority of cases $\log g$ obtained simultaneously with other parameters did not give consistent results, we decided to use only surface gravities determined as in Sect. 4 .

\section{The metallicities}

The metal abundance of the SPB and reference stars are presented in the third column of Tables 3, 4 and 5. The distribution of $[\mathrm{m} / \mathrm{H}]$ is presented in the histograms in Fig. 7. As one can see, slightly lower values of $[\mathrm{m} / \mathrm{H}]$ were found using the IUE data processed by the INES reduction package. The mean value of the metal abundance parameter for the SPB stars is equal to $-0.20 \pm 0.03$ and $-0.25 \pm 0.03$ dex for NEWSIPS and INES, respectively. For the reference stars $[\mathrm{m} / \mathrm{H}]$ amounts to $-0.18 \pm 0.03$ and $-0.25 \pm 0.03 \mathrm{dex}$. The mean values of the metallicities of the SPB stars are the same, within the errors, as for reference objects. The metallicities of the SPB stars determined from the IUE/NEWSIPS spectra range from -0.41 dex for HD 21071 to +0.03 dex for HD 74560. The extreme values determined from the IUE/INES data were obtained for HD 27396 and HD 74560; they are equal to -0.50 and -0.04 dex, respectively. The lowest values of $[\mathrm{m} / \mathrm{H}]$ of the SPB stars were obtained for HD 21071, HD 182255, HD 183133 and HD 27396 from both NEWSIPS and INES data. The metallicities of the reference stars extend to lower values. The lowest values occur for HD 115832 $(-0.42 \pm 0.07$ and $-0.58 \pm 0.11 \mathrm{dex}$ for NEWSIPS and INES data, respectively). The highest $[\mathrm{m} / \mathrm{H}]$ values were obtained for HD $105382(+0.24 \pm 0.02$ and $0.21 \pm 0.02$ dex $)$. However, HD 105382 is a peculiar star with a spot (Briquet et al. 2001).

\subsection{Comparison with previous work}

Out of all stars in our sample, three SPB (HD 147392, HD 160762 and HD 215573) and four reference stars

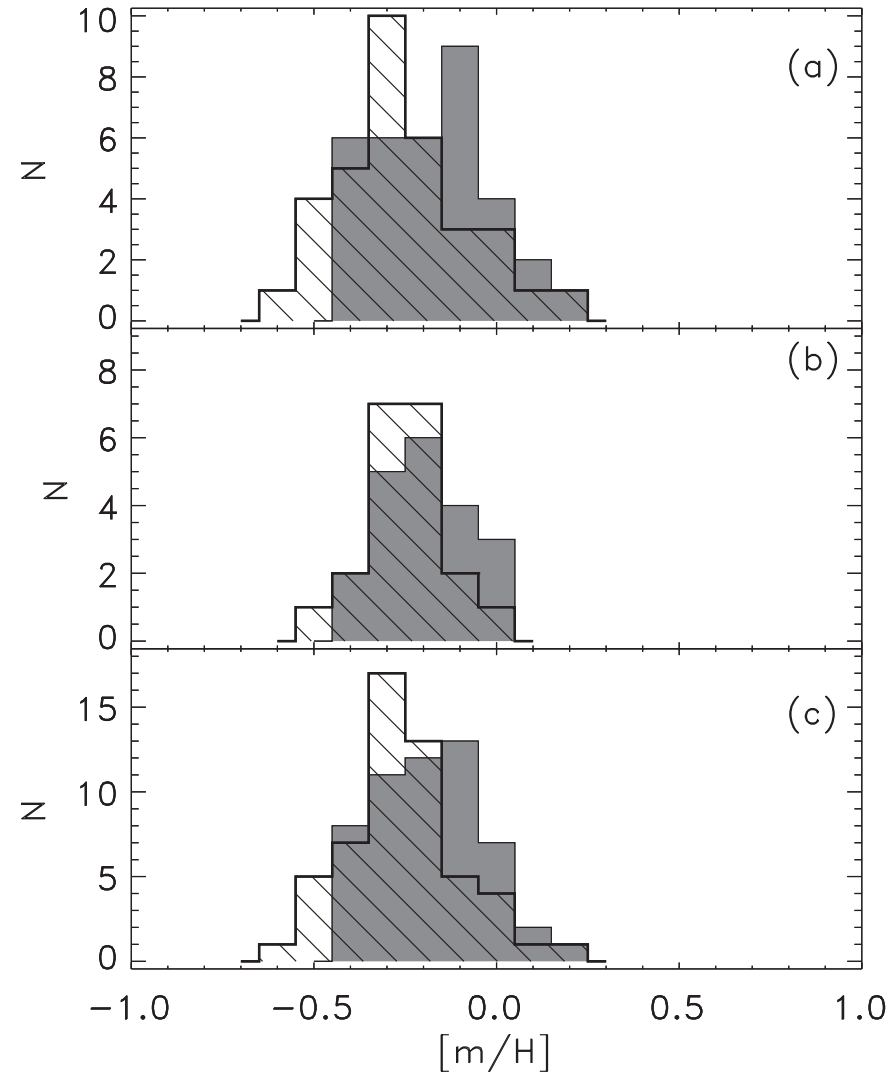

Fig. 7. The distribution of the metallicity for a) reference objects, b) SPB stars and c) all stars. The hatched histogram shows results for the IUE/INES data, while the gray histogram results for the IUE/NEWSIPS data.

(HD 17081, HD 135485, HD 184171, HD 209952) have been already objects of metallicity determinations.

For HD 147394 ( $\tau$ Her), Adelman (1977) determined $[\mathrm{Fe} / \mathrm{H}]=0.56 \mathrm{dex}$ from lines in the visual part of the spectrum. In two consecutive papers, this author found much lower values using optical lines, viz., $[\mathrm{Fe} / \mathrm{H}]=-0.13$ dex (Adelman 1986) and $[\mathrm{Fe} / \mathrm{H}]=-0.57$ dex (Adelman 1988). A detailed chemical composition of $\tau$ Her for several species was also derived by Adelman \& Philip (1996). In particular, these authors found $[\mathrm{FeII} / \mathrm{H}]=-0.32 \mathrm{dex}$ from the analysis of visual spectra. Smith \& Dworetsky (1993) obtained abundances of the iron group elements on the basis of the high-resolution IUE spectra and found the iron abundance of -0.14 dex. Morossi et al. (2002) determined $[\mathrm{m} / \mathrm{H}]=-0.05 \pm 0.07$ from the IUE/INES lowresolution spectra combined with the visual data. For $\tau$ Her we found the metal abundance of $-0.18 \pm 0.07$ and $-0.17 \pm 0.06$ for NEWSIPS and INES calibrations, respectively.

One of the well-studied SPB stars is HD 160762 ( $\iota$ Her). Kodaira \& Scholz (1970) obtained the abundance of iron from the analysis of lines in the visual part of the spectrum. They found $[\mathrm{FeII} / \mathrm{H}]=-0.79$ dex. Peters \& Aller (1970) obtained $[\mathrm{FeII} / \mathrm{H}]=-1.39 \mathrm{dex}$ from the lines in the visual part of the spectrum. Peters \& Polidan (1985) determined $-0.67 \mathrm{dex}$ from the UV and visual line spectra. Pintado \& Adelman (1993) obtained a similar value of -0.68 dex from visual lines alone. Adelman et al. (1993a) and Adelman \& Philip (1996) found 
-0.62 from the same range of wavelengths. Grigsby et al. (1996) determined $[\mathrm{FeII} / \mathrm{H}]=-0.47$ dex from high-resolution IUE spectra. From similar data, Proffitt \& Quigley (2001) obtained -0.37 dex. Morossi et al. (2002) found $[\mathrm{m} / \mathrm{H}]=$ $-0.21 \pm 0.04$ from low-resolution IUE/INES data. The metal abundances determined in this paper are consistent with Morossi et al. (2002) and are equal to $-0.19 \pm 0.06$ (IUE/NEWSIPS) and $-0.22 \pm 0.06$ (IUE/INES).

For HD 215573 ( $\xi$ Oct), Adelman et al. (1993b) found $[\mathrm{FeII} / \mathrm{H}]=-0.33$ dex from optical lines. Smith \& Dworetsky (1993) determined $[\mathrm{Fe} / \mathrm{H}]=-0.16 \mathrm{dex}$ from high-resolution IUE spectra. We obtained $[\mathrm{m} / \mathrm{H}]=-0.21 \pm 0.01$ from IUE/NEWSIPS and $-0.25 \pm 0.03$ from IUE/INES lowresolution data.

HD 17081 ( $\pi$ Cet) has been the object of iron abundance determination since late sixties. Wolff (1967) found $[\mathrm{Fe} / \mathrm{H}]=$ -0.40 dex from the optical part of the spectrum. A significantly different value of $[\mathrm{Fe} / \mathrm{H}]=0.70$ dex was obtained by Heacox (1979), also from the visual spectrum. Adelman (1984) and Adelman \& Fuhr (1985) determined, on the basis of optical lines, the iron abundance of -0.08 and $-0.05 \mathrm{dex}$, respectively. However, Adelman (1991) found $[\mathrm{FeII} / \mathrm{H}]=$ -0.29 dex. Similar values, $[\mathrm{FeII} / \mathrm{H}]=-0.28$ and -0.35 from the UV spectra and -0.29 from visual data were obtained by Adelman et al. (1993a). Smith \& Dworetsky (1993) determined $[\mathrm{Fe} / \mathrm{H}]=0.00$ dex from the co-added high-resolution IUE spectra. Nearly the same value $[\mathrm{Fe} / \mathrm{H}]=-0.10$ dex was obtained by Adelman (1998) from medium-resolution opticalspectra. The same value was obtained by Roby et al. (1999). We obtained slightly lower values, $[\mathrm{m} / \mathrm{H}]=-0.29 \pm 0.03$ from IUE/NEWSIPS and $-0.36 \pm 0.04$ from IUE/INES lowresolution data.

HD 135485 is a mid B-type star with a very sharp and prominent metal line spectrum. The first investigation of HD 135485 was done by Dufton (1973), who found $[\mathrm{Fe} / \mathrm{H}]=+1.9$ and +1.4 dex from moderate-resolution photographic spectra. The most recent investigation of metal content of HD 135485 was done by Trundle et al. (2001) on the basis of moderate- and low-resolution spectra covering the wavelength range 3745-6913 $\AA$. These authors found an enhancement of the iron group elements (FeII - III, CrII, MnII and NiII) of about $0.30 \mathrm{dex}$. We obtained $[\mathrm{m} / \mathrm{H}]=+0.13 \pm$ 0.09 from IUE/NEWSIPS and $+0.09 \pm 0.09$ from IUE/INES low-resolution data. These values indicate the excess of metals in HD 135485.

For HD 184171 (8 Cyg), Gies \& Lambert (1992) determined $[\mathrm{FeII} / \mathrm{H}]=-0.19$ dex, from optical lines. Adelman (1998) found $[\mathrm{FeII} / \mathrm{H}]=-0.33 \mathrm{dex}$ from the mediumresolution optical data. Proffitt \& Quigley (2001) obtained the iron abundance of -0.36 dex from co-added high-resolution IUE spectra. The metallicities determined in this paper are consistent with the earlier investigations and are equal to $[\mathrm{m} / \mathrm{H}]=$ $-0.23 \pm 0.08$ from IUE/NEWSIPS and $-0.21 \pm 0.08$ from IUE/INES low-resolution data.

For HD 209952 ( $\alpha$ Gru) only one metal abundance determination can be found in the literature. Fitzpatrick $\&$ Massa (1999) obtained $[\mathrm{m} / \mathrm{H}]=-0.35 \pm 0.05 \mathrm{dex}$ from IUE/NEWSIPS low-resolution spectra. We determined

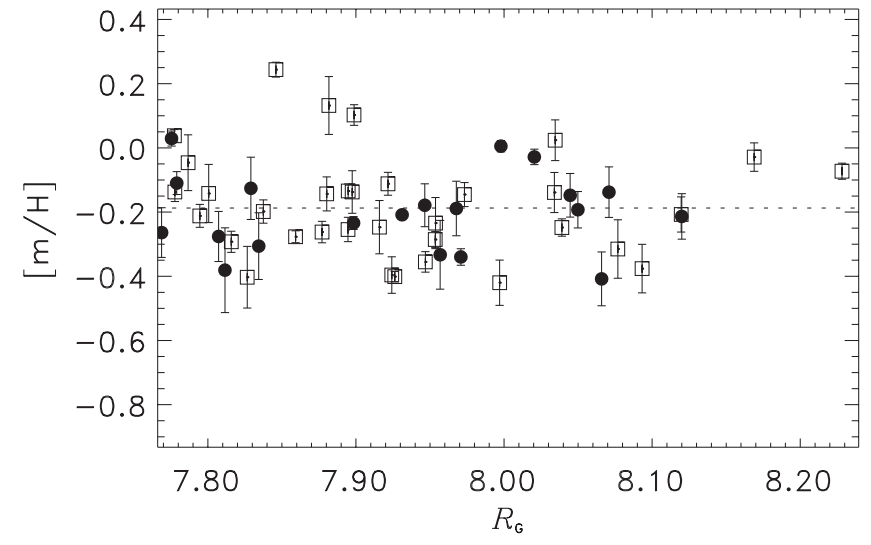

Fig. 8. The distribution of the metallicity parameter of the SPB (filled circles) and reference stars (squares) from the IUE/NEWSIPS data as a function of the galactocentric distance (in $\mathrm{kpc}$ ). The dashed line denotes the mean value of $[\mathrm{m} / \mathrm{H}]$ for all stars.

a higher metallicity values, equal to $[\mathrm{m} / \mathrm{H}]=-0.13 \pm 0.02$ from IUE/NEWSIPS and $-0.19 \pm 0.03$ from IUE/INES lowresolution data.

\subsection{Discussion of the parameter $[\mathrm{m} / \mathrm{H}]$}

Using the Hipparcos parallaxes (ESA 1997) of all analyzed stars, we calculated their galactocentric distances adopting the galactocentric distance of the Sun, $R_{\mathrm{G}, \odot}=7.9 \mathrm{kpc}$ (McNamara et al. 2000). The distribution of $[\mathrm{m} / \mathrm{H}]$ as a function of this distance is presented in Fig. 8. The stars are spread over a small distance of about $0.5 \mathrm{kpc}$ in the galactocentric distance, so that no clear indication of the metallicity gradient is seen. Nevertheless, we would like to add that even when taking into account the errors of $[\mathrm{m} / \mathrm{H}]$, some intrinsic scatter of the metallicity is certainly present. It is, however, even more important that the average metallicities of the SPB and the reference stars agree with each other and amount to about -0.20 dex.

Metal abundances of hot stars in the solar vicinity lower by about 0.20 dex than the solar value were reported by Gies \& Lambert (1992), Cunha \& Lambert (1994), Kilian (1992), Kilian et al. (1994), Daflon et al. (1999), Gummersbach et al. (1998) among others. These results were obtained from both high- and low-resolution data. An authors used Kurucz ATLAS models of stellar atmospheres. Metal abundances obtained in the present paper from the low-resolution IUE observations are consistent with these results.

The UV spectral region of the main-sequence B type stars is dominated by the iron lines (see, for example, Fitzpatrick \& Massa 1999). In addition to $\mathrm{Fe}$, the other iron-group elements like $\mathrm{Cr}, \mathrm{Mn}, \mathrm{Co}$ and Ni contribute to the UV opacity, but in a much lower degree. The Kurucz models use the reference solar Fe abundance equal to 0.001878 in the mass units, whereas the values adopted currently in OPAL (Iglesias et al. 1992; Iglesias \& Rogers 1996) and OP (Seaton et al. 1994) amount to 0.001338 and 0.001261 , respectively. In other words, the value of $[\mathrm{Fe} / \mathrm{H}]$ in the Kurucz's models is larger than in the OPAL and OP data. One can therefore expect that $[\mathrm{Fe} / \mathrm{H}]$ determined using the ATLAS9 models should be lower by 
about 0.12 dex in comparison with $[\mathrm{Fe} / \mathrm{H}]$ obtained on the basis of the OPAL or OP data (see also Fitzpatrick \& Massa 1999).

The metallicities of the SPB stars predicted by the theory of pulsations are not in contradiction with the values determined in this paper. Pamyatnykh (1999) adopted initial heavy element mixture after Grevesse \& Noels (1993) and showed that although the SPB instability strip shrinks with decreasing $Z$, it vanish for $Z$ as low as 0.006 , corresponding to [m/H] $\approx$ -0.50 dex. There is no SPB star with the metallicity lower than this limit.

\section{Conclusions}

In this paper we analyzed low-resolution IUE spectra of 20 SPB stars and 34 reference stars. The results of our investigation can be summarized as follows:

1. We derived stellar parameters $T_{\text {eff }},[\mathrm{m} / \mathrm{H}], \theta$ and $E(B-V)$. For some of the stars (HD 181558, HD 181133, HD 26326, HD 34798, HD 177863, HD 182255, HD 208057, HD 6417, HD 67536, HD 37332, HD 87015), effective temperatures were determined spectroscopically for the first time. For most our stars the metallicity parameter was never obtained before. Only for seven of them (HD 147394, HD 160762, HD 215573, HD 17081, HD 135485, HD 184171 and HD 209952) we can compare our values with the metal abundances obtained in a different way. We found a good consistency between metallicities determined in this paper and the literature values, even if the latter were obtained from the high-resolution spectra.

2. We derived metallicities simultaneously with the other stellar parameters using an algorithmic method recently proposed by Fitzpatrick \& Massa (1999). We also determined more accurate errors by using the bootstrap resampling method. This method was also used to obtain correlations between parameters. We found no correlations between metallicity and other parameters.

3. Metal abundances of the SPB stars predicted by the theory of pulsations are not in contradiction with the metallicities determined in this paper. The instability domain in the HR diagram disappears for $Z$ as low as 0.006 (which corresponds to $[\mathrm{m} / \mathrm{H}] \approx-0.50$ ). All metallicities we obtained for the SPB stars are higher than -0.50 dex.

4. We also show how the choice between the two methods of calibration and reduction of the IUE data, NEWSIPS and INES, influences the atmospheric parameters of the stars. It appears that different calibrations only slightly affect the metallicities we derived.

5 . The mean value of the metallicity parameter, $[\mathrm{m} / \mathrm{H}] \approx$ -0.20 was obtained for both the SPB and reference stars, regardless of the IUE calibration. This means that SPB stars do not differ from the other B-type stars as far as the metallicity is concerned. There must be another selection mechanism which causes that only some main-sequence B-type stars reach observable pulsations amplitudes.

Acknowledgements. I am grateful to Prof. H. Cugier and Prof. A. Pigulski for helpful discussions and comments on the manuscript. The detailed comments of the referee are acknowledged. I am grateful to Dr. A. Pamyatnykh for his calculations of the ZAMS stellar models.

\section{References}

Abt, H. A. 1988, ApJ, 331, 922

Abt, H. A., \& Levy, S. G. 1978, ApJS, 36, 241

Adelman, S. J. 1977, MNRAS, 181, 667

Adelman, S. J. 1984, A\&AS, 58, 585

Adelman, S. J. 1986, A\&AS, 64, 173

Adelman, S. J. 1988, MNRAS, 230, 671

Adelman, S. J. 1991, MNRAS, 252, 116

Adelman, S. J. 1998, MNRAS, 296, 856

Adelman, S. J., \& Fuhr, J. R. 1985, A\&A, 152, 434

Adelman, S. J., \& Philip, A. G. D. 1996, MNRAS, 282, 1181

Adelman, S. J., Cowley, C. R., Leckrone, D. S., Roby, S. W., \& Wahlgren, G. M. 1993a, ApJ, 419, 276

Adelman, S. J., Robinson, R. D., \& Wahlgren, G. M. 1993b, PASP, 105,327

Alekseeva, G. A., Arkharov, A. A., Galkin, V. D., et al. 1997, Baltic Astron., 6, 481

Aznar Cuadrado, R., \& Jeffery, C. S. 2001, A\&A, 368, 994

Babu, G. J., \& Singh, K. 1983, Annals. Statist., 11, 999

Balona, L. A. 1994, MNRAS, 268, 119

Bessel, M. S. 1979, PASP, 91, 589

Bevington, P. R. 1969, in Data Reduction and Error Analysis for the Physical Science (New York: McGraw-Hill)

Bogges, A., Carr, F. A., Evans, D. C., et al. 1978, Nature, 275, 372

Breger, M. 1976, ApJ, 32, 7

Briquet, M., Aerts, C., \& De Cat, P. 2001, A\&A, 366, 121

Chapellier, E., Sadsaound, H., Valtier, J. C., et al. 1998, A\&A, 331, 1046

Chapellier, E., Mathias, P., Le Contel, J.-M., et al. 2000, A\&A, 362, 198

Code, A. D., Bless, R. C., Davis, J., \& Brown, R. H. 1976, ApJ, 203, 417

Cunha, K., \& Lambert, D. L. 1994, ApJ, 426, 170

Daflon, S., Cunha, K., \& Becker, S. R. 1999, ApJ, 522, 950

Daszyńska, J., Niemczura, E., \& Cugier, H. 2002, Adv. Space Res., 31,387

De Cat, P., Aerts, C., De Ridder, J., et al. 2000, A\&A, 355, 1015

De Cat, P. 2001, Ph.D. Thesis, Leuven, Belgium

De Ridder, J., Gordon, K. D., Mulliss, C. L., \& Aerts, C. 1999, A\&A, 341,574

Dufton, P. L. 1973, A\&A, 28, 267

Dziembowski, W. A., \& Pamyatnykh, A. A. 1993, MNRAS, 262, 204

ESA 1997, The Hipparcos and Tycho Catalogues

Fitzpatrick, E. L. 1999, PASP, 111, 63

Fitzpatrick, E. L., \& Massa, D. 1999, ApJ, 525, 1011

Gahart, M. P., Smith, M. A., Levay, K. L., \& Thompson, R. W. 1997, IUE NEWSIPS Information Manual, Version 2.0

Gahm, G. F., Ahlin, P., \& Lindroos, K. P. 1983, A\&AS, 51, 143

Gautchy, A., \& Saio, H. 1993, MNRAS, 262, 213

Gies, D. R., \& Lambert, D. L. 1992, ApJ, 387, 673

Gonzáles-Riestra, R., Cassatella, A., \& Wemsteker, W. 2001, A\&A, 373, 730

Gray, R. O. 1998, ApJ, 116, 482

Grevesse, N., \& Noels, A. 1993, in ed. N. Pratzo, E. Vangioni-Flam, $\&$ M. Casse, Origin and Evolution of the Elements, 15

Grigsby, J. A., Mulliss, C. L., \& Baer, G. M. 1996, PASP, 108, 953

Gummersbach, C. A., Kaufer, D. R., Schäfer, D. R., Szeifert, T., \& Wolf, B. 1998, A\&A, 338, 883

Hartkopf, W. I., Mason, B. D., McAlister, H. A., et al. 2000, AJ, 119, 3084

Heacox, W. D. 1979, ApJS, 41, 475

Hube, D. P., \& Aikman, G. C. L. 1991, PASP, 103, 49

Iglesias, C. A., Rogers, F. J., \& Wilson, B. G. 1992, ApJ, 397, 717 
Iglesias, C. A., \& Rogers, F. J. 1996, ApJ, 464, 943

Jerzykiewicz, M., \& Molenda-Żakowicz, J. 2000, Acta. Astron., 50, 369

Kilian, J. 1992, A\&A, 262, 171

Kilian, J., Montenbruck, O., \& Nissen, P. E. 1994, A\&A, 284, 437

Kobi, D., \& North, P. 1990, A\&AS, 85, 999

Kodaira, K., \& Scholz, M. 1970, A\&A, 6, 93

Koen, C. 2001, MNRAS, 321, 44

Kurucz, R. 1996, CD-ROM, No. 13 and 19

Künzli, M., North, P., Kurucz, R. L., \& Nicolet, B. 1997, A\&A, 122, 51

Lawson, W. A., Clark, M., \& Cottrell, P. L. 1994, MNRAS, 266, 740

Lutz, T. E., \& Kelker, D. H. 1973, PASP, 85, 573

Maceroni, C., \& Ruciński, S. M. 1997, PASP, 109, 782

Masuda, S., \& Hirata, R. 2000, A\&A, 356, 209

McNamara, D. H., Madsen, J. B., Barnes, J., \& Ericksen, B. F. 2000, PASP, 112, 202

Mermilliod, J. C., Mermilliod, M., \& Hauck, B. 1997, A\&AS, 124, 349

Molenda-Żakowicz, J. 2002, Ph.D. Thesis, Wrocław University, Poland

Moon, T. T., \& Dworetsky, M. M. 1985, MNRAS, 217, 305

Morossi, C., Di Marcantonio, P., Franchini, M., Malagnini, M. L., \& Chavez, M. 2002, ApJ, 577, 377

Napiwotzki, R., Schönberner, D., \& Wenske, V. 1993, A\&A, 268, 653

Nichols, J. S., \& Linsky, J. L. 1996, AJ, 111, 517

Niemczura, E., Daszyńska, J., \& Cugier, H. 2002, Adv. Space Res., 31, 399

North, P., \& Nicolet, B. 1990, A\&A, 228, 78

North, P., \& Paltani, S. 1994, A\&A, 288, 155
Pamyatnykh, A. A. 1999, Acta Astron., 49, 119

Peters, G. J., \& Aller, L. H. 1970, ApJ, 159, 525

Peters, G. J., \& Polidan, R. S. 1985, in Calibration of Fundamental Stellar Properties, ed. D. S. Hayes, et al., IAU Symp., 111, 417 (Dordrecht: Reidel)

Pintado, O. I., \& Adelman, S. J. 1993, MNRAS, 264, 63

Press, W. H., Teukolsky, S. A., \& Vetterling, W. T. 1992, Numerical Recipes in Fortran, 2nd ed. (Cambridge and New York: Cambrige University Press)

Proffitt, C. R., \& Quigley, M. F. 2001, ApJ, 548, 429

Ribas, I., Jordi, C., Torra, J., \& Giménez, A. 1997, A\&A, 327, 207

Roby, S. W., Leckreone, D. S., \& Adelman, S. J. 1999, ApJ, 524, 974

Rodríguez Pascual, P. M., González-Riestra, R., Schartel, N., \& Wemsteker, W. 1999, A\&AS, 139, 183

Schartel, N., \& Skillen, I. 1998, in UV Astrophysics, Beyond the IUE Final Archive, ed. W. Wamsteller, \& R. Gonzalez Riestra (ESTEC: Noordwijk), 735

Seaton, M. J., Yan, Y., Mihalas, D., \& Pradhan, A. K. 1994, MNRAS, 266, 805

Smith, K. C., \& Dworetsky, M. M. 1993, A\&A, 274, 335

Tokovinin, A. A. 1997, A\&AS, 124, 75

Trundle, C., Dufton, P. L., Rolleston, W. R. J., et al. 2001, MNRAS, 328, 291

Waelkens, C. 1991, A\&A, 246, 468

Waelkens, C. 1987, in Lecture Notes in Physics 274, ed. A. N. Cox, W. M. Sparks, \& S. G. Starrfield, 75 (Springer-Verlang)

Waelkens, C., \& Rufener, F. 1985, A\&A, 152, 6

Waelkens, C., Aerts, C., Kestens, E., Grenon, M., \& Eyer, L. 1998, A\&A, 330, 215

Wolff, S. C. 1967, ApJS, 15, 21 